\title{
Manifestation of the quantum size effect in transmission resonance
}

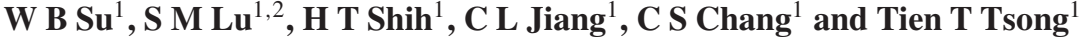 \\ ${ }^{1}$ Institute of Physics, Academia Sinica, Taipei, Taiwan, Republic of China \\ ${ }^{2}$ Department of Physics, National Taiwan University, Taipei, Taiwan, Republic of China \\ E-mail: wbsu@phys.sinica.edu.tw
}

Received 17 April 2006, in final form 22 May 2006

Published 23 June 2006

Online at stacks.iop.org/JPhysCM/18/6299

\begin{abstract}
The transmission probability of the free electron scattered by the quantum well in metal film may reveal the phenomenon of resonance. This transmission resonance, appearing above the vacuum level, can be probed by scanning tunnelling spectroscopy (STS). In this paper, STS is used to observe the transmission resonance on $\mathrm{Ag}$ films grown on $\mathrm{Si}(111) 7 \times 7$ surfaces. The transmission resonance appears at different bias voltages in the spectra depending on the film thickness; its energy level moves toward the vacuum level with increasing film thickness. When the film is thick enough, transmission resonance can occur twice in the biasing voltage range, and the energy separation between the resonances decreases with increasing film thickness. The existence of transmission resonance affects the contemporary standing wave states (SWS) and, in general, pushes the following SWS to higher energies.
\end{abstract}

\section{Introduction}

When the thickness of a metal film is comparable to the electron de Broglie wavelength, electrons in the film as well as those being transmitted through the film can manifest the quantum size effect (QSE). For the former, electrons are confined in a quantum well of the metal film and form quantized standing wave states along the surface normal. This kind of QSE can cause the properties of the film to differ significantly from those of the bulk, such as the oscillation behaviour with film thickness in electrical resistivity [1] and interlayer spacing [2, 3], as well as the manifestation of the quantum well states in the electronic structure [4-6]. This QSE is also believed to be a major driving force for the formation of atomically flat metal films on semiconductor surfaces [7-9]. For the latter, the electrons transmitted through the film, the electron QSE appears above the vacuum level and is attributed to the interference of the electron waves, which are reflected from the film surface and the film-substrate interface. With some specific energy, the electron can totally penetrate the film, and this is termed the 
transmission resonance [10-12]. Recently Chung et al have demonstrated that the thickness of Ag films grown on W(110) can be discriminated by probing the resonance peaks using low energy electron microscopy (LEEM) [13]. The first observation of transmission resonance by scanning tunnelling spectroscopy (STS) was made by Kubby et al on a $\sqrt{3} \times \sqrt{3} \mathrm{Sn} / \mathrm{Si}(111)$ surface $[14,15]$. As transmission resonance has been observed in the $\mathrm{Ag} / \mathrm{W}(110)$ system [10], it can be expected that it would also appear in the $\mathrm{Ag} / \mathrm{Si}(111) 7 \times 7$ system. In this report, it is demonstrated that by utilizing STS the transmission resonance can indeed be revealed in the tunnelling spectra of $\mathrm{Ag}$ films grown on $\mathrm{Si}(111) 7 \times 7$. As film thickness increases the energy levels of transmission resonance obviously shift toward the vacuum level, the separation between them subsequently being reduced. These thickness-dependent trends are consistent with the predictions of quantum theory.

\section{Experimental details}

For this experiment a $\mathrm{Si}(111) 7 \times 7$ surface was obtained by annealing the sample to $1200^{\circ} \mathrm{C}$ and then slowly cooling it to room temperature. Silver was deposited onto the $\mathrm{Si}(111) 7 \times 7$ surface at room temperature with a flux of $0.26 \mathrm{ML}$ per minute. The vacuum was kept below $2 \times 10^{-10}$ Torr during deposition. It is known that flat silver films with the (111) face can be grown on $\mathrm{Si}(111) 7 \times 7$ at room temperature [16]. After deposition, the sample was transferred to a home-built STM in which the sample was cooled to $109 \mathrm{~K}$. The electronic structures of the Ag films were probed using $Z-V$ spectroscopy. In the $Z-V$ measurement, the tip trajectory was recorded with an active feedback, while the sample bias was ramped from 2 to $9 \mathrm{~V}$. A modulation of the sample voltage of $30 \mathrm{mV}$ at a frequency of $5 \mathrm{kHz}$ is added to the sample bias. As the frequency of modulation is too high for the feedback circuit to respond, the tunnelling current contained a $5 \mathrm{kHz}$ component. This current modulation can be extracted by a lock-in amplifier. When a $Z-V$ spectrum is acquired, the signal from the lock-in amplifier is recorded simultaneously to obtain a spectrum similar to the $\mathrm{d} Z / \mathrm{d} V-V$ curve.

\section{Results and discussion}

Figure 1(a) shows a typical STM topography image of an Ag film prepared at room temperature with a coverage of 3.6 ML. At this coverage, about 0.35 ML of Ag were consumed to wet the $\operatorname{Si}(111) 7 \times 7$ surface [16] and the rest grew into flat films with the (111) face. The growth is not layer by layer; thus, several film thicknesses can often coexist. Film thickness can be accurately determined by measuring the height of the film against the exposed wetting layer. STS is used to take $Z-V$ spectra on films of different thicknesses. The black curve in figure 1(b) shows such a spectrum, taken on a film of nine atomic layers above the silicon substrate. For comparison, the spectrum is also acquired on the (111) surface of an Ag crystal, drawn as the grey curve in figure 1(b). The two curves are similar and reveal step-like features that signify the onset of a standing wave state (SWS) between the tip and sample [17, 18]. They also correspond to the numbered peak features in the $\mathrm{d} Z / \mathrm{d} V-V$ curves shown in figure 1(c). The SWS arises from the interference of the electron wave emitted from the tip and that reflected from the surface of the film. It thus adopts a nature similar to that of a bound state. Beside these peaks, two additional bumps marked by black downward arrows are observed in the curve associated with the film. However, these do not appear in the spectrum of the Ag crystal, indicating that the bump feature is specific to the Ag thin films. Figure 2(a) displays the spectra obtained by the lock-in technique, with the active feedback on the films containing 9-11 atomic layers (indicated by the number in parentheses). There, arrows mark the bump features appearing in the curves of different films, and it is obvious that the energy separation between the bump 


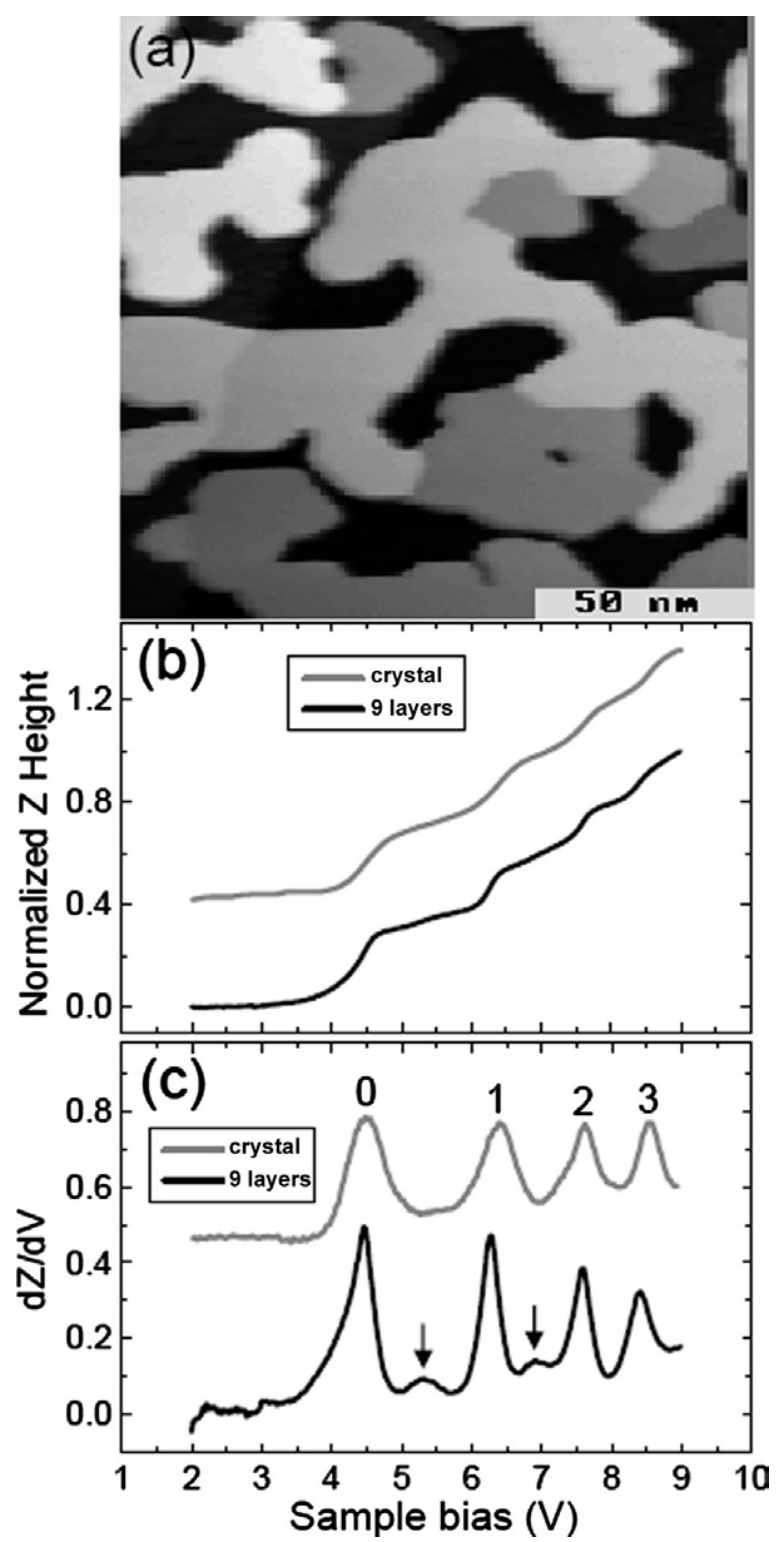

Figure 1. (a) The growth of flat $\mathrm{Ag}$ films on $\mathrm{Si}(111) 7 \times 7$ surface at room temperature at the coverage of $3.6 \mathrm{ML}$. The image size is $150 \times 150 \mathrm{~nm}^{2}$. (b) $Z-V$ spectra measured on the 9-layer Ag film (black curve) and crystal $\operatorname{Ag}(111)$ surface (grey curve). (c) $\mathrm{d} Z / \mathrm{d} V-V$ curves directly differentiated from $Z-V$ spectra in (b).

features decreases with increasing film thickness. Although the association of these bumps with film thickness is quite apparent, it does not have the same physical origin as that which is understood for conventional QSE. The conventional QSE involves bound states originating from the boundary confinement, yet the energy levels of the bump features are all located above the vacuum level (referring to the work function of the Ag film on $\mathrm{Si}(111)$ being $4.41 \mathrm{eV} \mathrm{[19]).}$ They derive from the electron waves scattered by a potential well. 

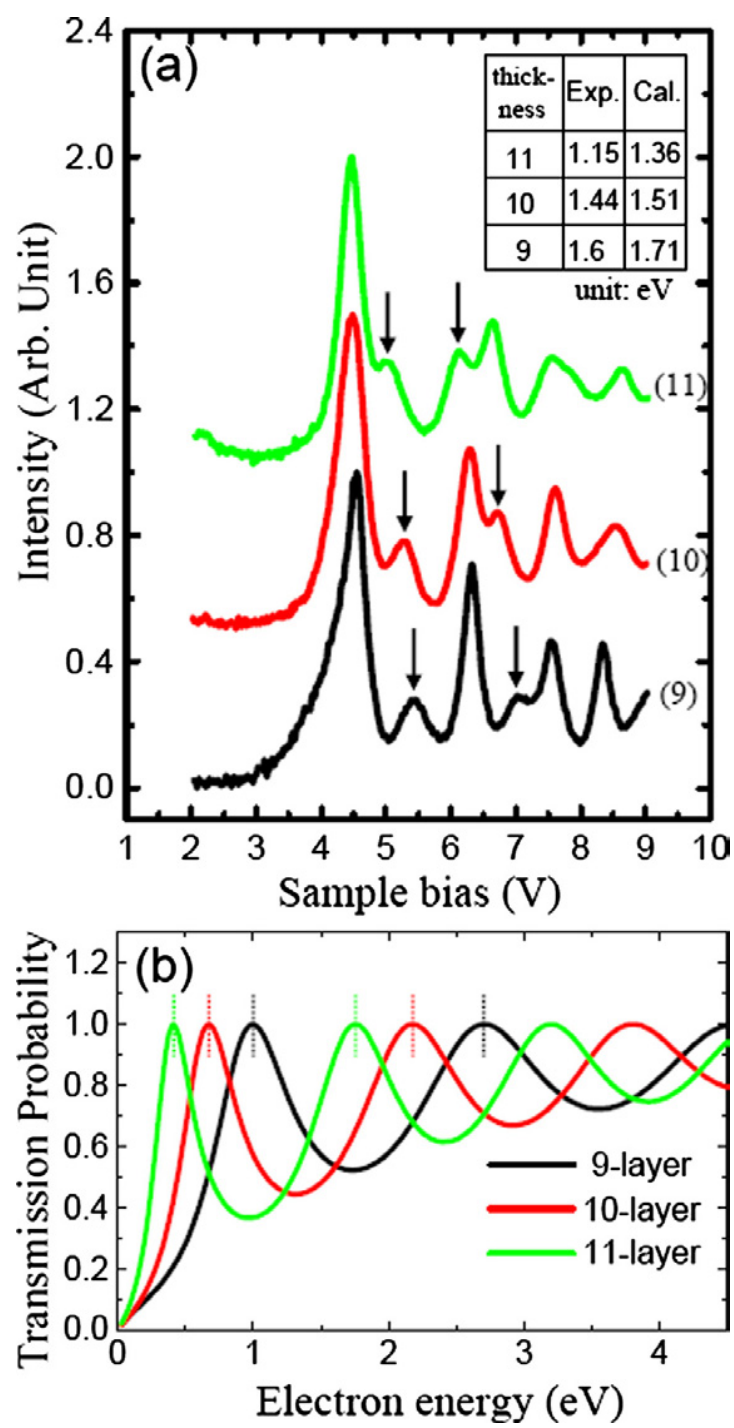

Figure 2. (a) Spectra acquired on 9-11-layer films by lock-in technique with the feedback kept active. Numbers in parentheses indicate the film thickness. (b) Calculated curves of transmission probability as a function of electron energy for 9-11-layer films. Dashed lines indicate energy levels of transmission resonance.

(This figure is in colour only in the electronic version)

According to quantum mechanics, the probability for a free electron being transmitted through a square potential well obeys the following equation [20]:

$$
1 / T=1+V^{2} \sin ^{2}(k t) / 4 E(E+V),
$$

where $T$ is the transmission probability, $E$ is the energy of the incident electron, $V$ is the depth of the potential well, $t$ is the width of the well, $k$ is the wavevector of electrons in the well and

$$
\hbar^{2} k^{2} / 2 m=E+V \text {. }
$$

In the current system, the thin Ag film grown on the Si substrate acts like a potential well to the 


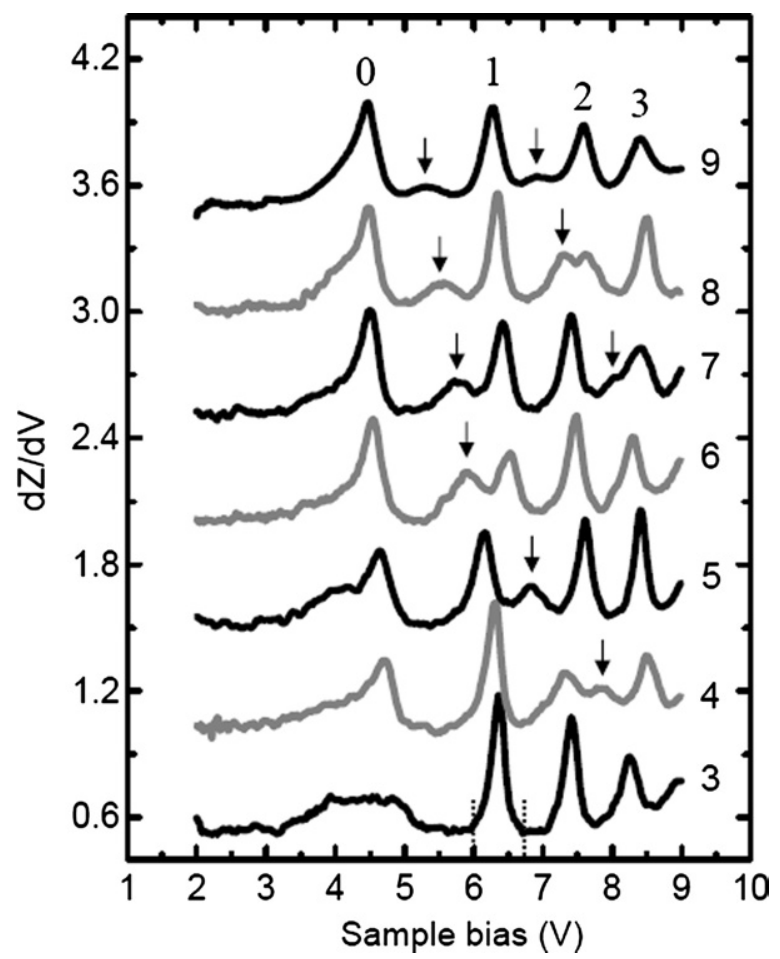

Figure 3. Spectra acquired on 3-9-layer films. Numbers at the right-hand sides of spectra mark atomic layers of the thickness. Arrows mark the positions of transmission resonance. Numbers at the top mark the standing wave states in the spectrum for each thickness.

electrons emitted from the tip. Figure 2(b) shows calculated curves of transmission probability as a function of electron energy for the films of 9-11 layers using equation (1). The bulk values for Ag crystal are adapted here, i.e. Fermi energy of $8 \mathrm{eV} \mathrm{[10]} \mathrm{for} V$ and $2.5 \AA$ for interlayer spacing along the (111) direction. Each calculated curve exhibits an oscillatory aspect, indicating that in general electrons can be transmitted and reflected at the potential well with different weighted probabilities. Nevertheless, at some specific energy levels (marked by dashed lines) electrons can penetrate the film completely, resulting in transmission resonance. The energy levels of transmission resonance move toward the vacuum level with increasing film thickness. This is consistent with the bump features shown in figure 2(a). The calculated (cal.) values of the energy separation between the first two transmission resonances are tabulated in figure 2(a) - these decrease with increasing film thickness and qualitatively agree with the experimental (exp.) measurements. It can thus be concluded that the bump features result from transmission resonance.

Figure 3 shows the $\mathrm{d} Z / \mathrm{d} V-V$ spectra acquired on 3- to 9-layer films. The number at the right-hand side of each curve denotes the film thickness. In each spectrum there appear four SWS peaks, marked by numbers, and one transmission resonance bump, marked by an arrow. Beyond a film thickness of six atomic layers a second transmission resonance begins to be observed. In some circumstances it is difficult to differentiate the bump from the peaks; for instance, the spectral intensities of SWS2 and the transmission resonance are about the same in the spectrum of the 8-layer film. However, the peak position of the SWS can be adjusted by tuning the tunnelling current [15], and in this way, the quick alteration in the position and 


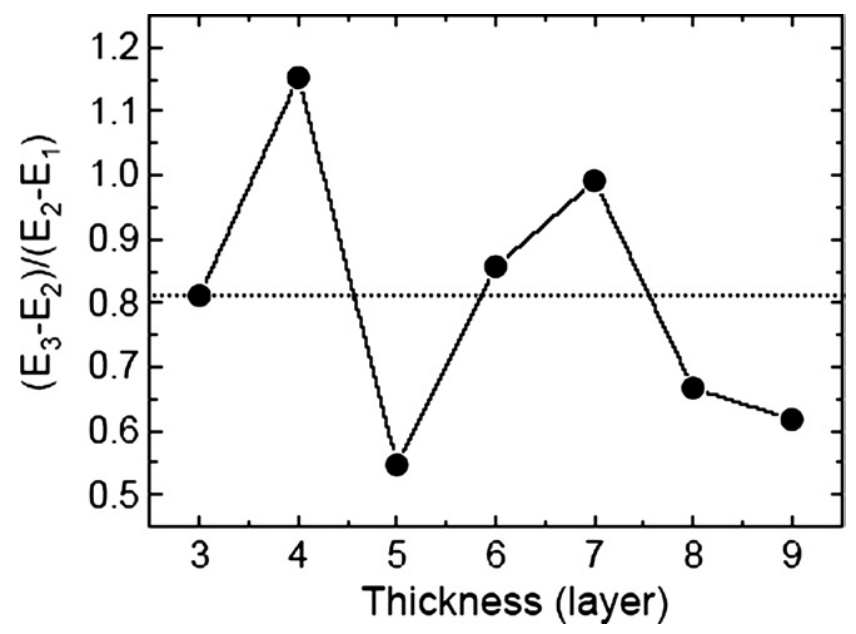

Figure 4. Ratio of $\left(E_{3}-E_{2}\right) /\left(E_{2}-E_{1}\right)$ obtained from the spectrum for each thickness in figure 3 . The dashed line indicates the value of 0.812 .

intensity of the SWS can be spotted and thus the transmission resonance bump can immediately be identified. The thickness-dependent behaviour shown in figure 3 is consistent with that of figure 2(a), i.e. as film thickness increases, the energy levels of transmission resonance move toward the vacuum level and their separation decreases. In addition, SWS1 in the spectrum of the 3-layer in figure 3 is a peak, and its onset and end (marked by dashed lines) can be precisely determined. The width of this peak can therefore be measured, and is about $0.7 \mathrm{eV}$.

Both standing wave states (SWS) acquiring bound state nature and transmission resonances are found in these spectra. Though they have different physical natures, they are very sensitive to what happens to the electrons at the film surface. The SWS relies much on the strong reflection of the electrons at the surface, while the appearance of the bump features is dependent on the transparency of the film to the incoming electrons. Hence, the interplay between the transmission resonance and SWS is worth further exploration. Kubby et al have demonstrated on a $\sqrt{3} \times \sqrt{3} \mathrm{Sn} / \mathrm{Si}(111)$ surface that the manifestation of transmission resonance can displace higher order SWS to a higher energy level [14]. In this paper this phenomenon is investigated using Ag films of different thicknesses in a more systematic way. Before proceeding, it should be noted that there is no sign of transmission resonance in the spectrum of the 3-layer film in figure 3, and therefore this spectrum will be used as a reference. The ratio of the energy separations between the SWS numbered 1, 2, 3, i.e. $\left(E_{3}-E_{2}\right) /\left(E_{2}-E_{1}\right)$, provides a guideline to measure any displacement of the SWS due to the appearance of transmission resonance. Figure 4 shows the experimentally obtained ratio of $\left(E_{3}-E_{2}\right) /\left(E_{2}-E_{1}\right)$ from the spectrum of each thickness in figure 3. It is clear that the ratio values scatter from that of 0.812 for the 3-layer film. The one closest to the reference is that of the 6-layer film, in which transmission resonance falls between SWS0 and SWS1, so the higher order SWS are all pushed to higher values but the ratio of energy separations is less affected. This will not be the case if the transmission resonance appears between SWS1 and SWS2 or SWS2 and SWS3. In the spectra of 5-, 8- and 9-layer films, one can see a transmission resonance existing between SWS1 and SWS2, causing a widening of the energy separation between them and hence a reduction in ratio. On the other hand, transmission resonance occurs between SWS2 and SWS3 in the spectra of 4- and 7-layer films and shifts SWS3 to a higher energy, as shown in figure 3. Therefore, the separation ratios for the 4- and 7-layer films are above the reference value. 
These results thus strongly suggest that the displacement of SWS by the transmission resonance should be a universal phenomenon.

\section{Conclusions}

In summary, the quantum phenomenon of transmission resonance on Ag films has been studied by scanning tunnelling spectroscopy. The characteristics of transmission resonance as a function of the film thickness can be understood using the model of free electrons scattered by a one-dimensional quantum well. The interplay between transmission resonance and standing wave states was also investigated, and it was found that the displacement of higher order SWS by the transmission resonance is a universal phenomenon.

\section{Acknowledgments}

The authors would like to acknowledge M Y Chou for pointing out that our spectra reveal information on transmission resonance, C M Wei for helpful discussions, and C S Kuo, C Y Lin, and $\mathrm{C} \mathrm{H}$ Hsieh for assisting in the construction of the STM. This work was supported by the National Science Council, Academia Sinica, and the project of Academic Excellence of the Ministry of Education of Taiwan.

\section{References}

[1] Jalochowski M, Knoppe H, Lilienkamp G and Bauer E 1992 Phys. Rev. B 464693 Jalochowski M and Bauer E 1988 Phys. Rev. B 385272

[2] Crottini A, Cvetko D, Floreano L, Gotter R, Morgante A and Tommasini F 1997 Phys. Rev. Lett. 791527

[3] Su W B, Chang S H, Jian W B, Chang C S, Chen L J and Tsong T T 2001 Phys. Rev. Lett. 865116

[4] Miller T, Samsavar A, Franklin G E and Chiang T-C 1988 Phys. Rev. Lett. 611404

[5] Schmid M, Hebenstreit W, Varga P and Crampin S 1996 Phys. Rev. Lett. 762298

[6] Altfeder I B, Matveev K A and Chen D M 1997 Phys. Rev. Lett. 782815

[7] Smith A R, Chao K J, Niu Q and Shih C K 1996 Science 273226

[8] Zhang Z, Niu Q and Shih C K 1998 Phys. Rev. Lett. 805381

[9] Budde K, Abram E, Yeh V and Tringides M C 2000 Phys. Rev. B 61 R 10602

[10] Jonker B T, Bartelt N C and Park R L 1983 Surf. Sci. 127183

[11] Bauer E 1994 Rep. Prog. Phys. 57895

[12] Altman M S, Chung W F and Liu C H 1998 Surf. Rev. Lett. 51129

[13] Chung W F, Feng Y J, Poon H C, Chan C T, Tong S Y and Altman M S 2003 Phys. Rev. Lett. 90216105

[14] Kubby J A, Wang Y R and Greene W J 1990 Phys. Rev. Lett. 652165

[15] Kubby J A and Greene W J 1993 Phys. Rev. B 4811249

[16] Sobotík P, Ošt'ádal I, Mysliveček J, Jarolímek T and Lavický F 2001 Surf. Sci. 482-485 797

[17] Binnig G, Frank K H, Fuchs H, Garcia N, Reihl B, Rohrer H, Salvan F and Williams A R 1985 Phys. Rev. Lett. $\mathbf{5 5} 991$

[18] Becker R S, Golovchenko J A and Swartzentruber B S 1985 Phys. Rev. Lett. 55987

[19] Thanailakis A 1975 J. Phys. C: Solid State Phys. 8655

[20] Liboff R L 1980 Introductory Quantum Mechanics (Reading, MA: Addison-Wesley) p 224 